\title{
CODEX: measuring the acceleration of the universe and beyond
}

\author{
Luca Pasquini $^{1}$, S. Cristiani ${ }^{2}$, H. Dekker ${ }^{1}$, M. Haehnelt ${ }^{3}$, P. Molaro ${ }^{2}$, \\ F. Pepe ${ }^{4}$, G. Avila ${ }^{1}$, B. Delabre ${ }^{1}$, S. D'Odorico ${ }^{1}$, J. Liske ${ }^{1}$, \\ P. Shaver ${ }^{1}$, P. Bonifacio ${ }^{2}$, S. Borgani ${ }^{2}$, V. D'Odorico ${ }^{2}$, E. Vanzella ${ }^{2}$, \\ F. Bouchy ${ }^{4}$, M. Dessauges ${ }^{4}$, C. Lovis ${ }^{4}$, M. Mayor ${ }^{4}$, D. Queloz ${ }^{4}$, \\ S. Udry ${ }^{4}$, M. Murphy ${ }^{3}$, M. Viel ${ }^{3}$, A. Grazian ${ }^{5}$, S. Levshakov ${ }^{6}$, \\ L. Moscardini ${ }^{7}$, T. Wiklind ${ }^{8}$ \& S. Zucker ${ }^{9}$ \\ ${ }^{1}$ European Southern Observatory, Garching, Germany \\ email: lpasquin@eso.org \\ ${ }^{2}$ INAF-Osservatorio Astronomico di Trieste, Italy \\ ${ }^{3}$ Institute of Astronomy, Cambridge University, United Kingdom \\ ${ }^{4}$ Observatoire de Geneve, Switzerland \\ ${ }^{5}$ INAF-Osservatorio Astronomico di Roma, Italy \\ ${ }^{6}$ Ioffe Physical-Technical Institute, St. Petesburg, Russian Federation \\ ${ }^{7}$ University of Bologna, Italy \\ ${ }^{8}$ ESA-STSci, Baltimore, USA \\ ${ }^{9}$ Weizmann Institute of Science, Tel Aviv, Israel
}

\begin{abstract}
The combination of the collecting power of an ELT with an ultra-stable high resolution spectrograph opens up the possibility to measure for the first time directly the dynamical effect of the acceleration of the Universe. CODEX will also provide unique opportunities for advance in many other branches of astrophysics. The CODEX design is based on an array of several identical spectrographs. It is highly modular and can be easily adapted to a large range of sky apertures and telescope diameters. CODEX is designed to work as a seeing limited instrument. The requirements for the telescope are moderate and clearly identified.
\end{abstract}

Keywords. Quasars: absorption lines, Techniques: radial velocities, Cosmology: observations

\section{The main experiment}

The expansion of the Universe is one of the pillars of the cosmological standard model. The change of the expansion rate means that an object at fixed coordinate distance which has a redshift $z_{0}$ at time $\mathrm{T}_{0}$ will have a slightly different redshift $z_{1}$ at a later time $\mathrm{T}_{1}$. This change of redshift is tiny but in principle measurable by waiting a long enough time.

The experiment proposed is conceptually very simple: by taking spectra of high redshift objects with a time interval of several years, it is possible to detect and use the wavelength shifts of spectral features emitted at high redshift to probe directly the change of the expansion rate of the Universe.

Within General Relativity (GR) a homogeneous and isotropic Universe is be described by the Friedmann-Robertson-Walker (FRW) metric. The relevant quantitity for the change of the redshift with time is the evolution of the Hubble parameter $\mathrm{H}$, which is easily described as: 


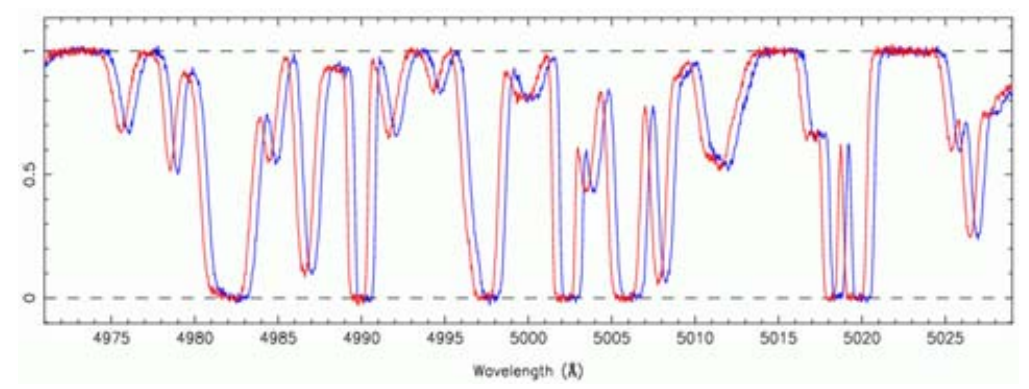

Figure 1. Simulated spectra of the Ly $\alpha$ forest of a QSO taken with a time interval of $10^{7} \mathrm{yrs}$. The shift due to the cosmic acceleration is clearly visible.

$$
H=H_{0}\left[\Omega_{\text {mat }}\left(a_{0} / a\right)^{3}+\Omega_{R}\left(a_{0} / a\right)^{4}+\Omega_{d e}\left(a_{0} / a\right)^{3(1+w)}+\left(1-\Omega_{t o t}\right)\left(a_{0} / a\right)^{2}\right]^{1 / 2}
$$

where $\Omega_{\text {tot }}=\Omega_{\text {mat }}+\Omega_{R}+\Omega_{d e} \cdot \Omega_{\text {mat }}+\Omega_{R}+\Omega_{d e}$ are the energy density of matter, radiation and dark energy respectively.

Recent observations, most noticeably the WMAP observations of the CMB Spergel et al. (2001), the search for high redshift supernovae (Riess et al. (1998), Perlmutter et al. (1999)) and clustering of galaxies provide a convergent picture which indicates that $\Omega_{t o t} \sim 1$ and that the dark energy is compatible with beeing due to a cosmological constant term in the Einstein equation with $\Omega_{d e} \sim 0.7$.

The above-mentioned constraints on the cosmological parameters are basically geometric in nature, as they mainly constrain the angular diameter distance of the last scattering surface (CMB) and the luminosity distance at moderate redshifts (SNae). A direct measure of the dynamical effect of the dark energy would be an important consistency check for the theory of General Relativity in its standard form.

The concept of the measurement is shown in Figure 1, which shows two spectra of the same Ly $\alpha$ absorption system taken at $10^{7}$ years interval. The shift is largely exxagerated and thus clearly visibile. The idea of performing this measurement was already analyzed by Sandage (1962), concluding that the expected shifts were too small to be detected. Indeed, as discussed, e.g. by Pasquini et al. (2005), for the concordance cosmological model the shift expected is of the order of $0.5 \mathrm{~cm} \mathrm{~s}^{-1} \mathrm{yr}^{-1}$ in the redshift range $z=2-5$. The measurement of such small wavelength shift provides strong constraints for the selection of suitable targets. Many promising candidates as, for instance, masers and molecular absorptions, reside in deep potential wells. The wavelength shifts will thus be dominated by peculiar accelerations which are larger than the cosmic signal. Fortunately, this is not the case for the absorption features of the Ly $\alpha$ forest in the spectra of high-redshift QSOs which observations and numerical simulations have clearly shown to reside in shallow potentials and to beautifully follow the cosmic flow. We have performed extensive simulations to demonstrate that the detection of the cosmic acceleration is indeed feasible. The signal should be detectable for observations taken with an interval of 10 years if the lines are resolved and an adequate $\mathrm{S} / \mathrm{N}$ ratio is obtained (a cumulative $\mathrm{S} / \mathrm{N}$ ratio of the order of 13500 for a $0.0125 \AA$ pixel). Figure 2 shows the results of a full simulation, corresponding to 2000 hours of integration on a 16.5 magnitude QSO with an 80 meter telescope and an overall efficiency equal to the one of UVES at the VLT. The signal is detected at high significance. Such a high cumulative $\mathrm{S} / \mathrm{N}$ ratio can be obtained by adding the results for a sample of QSOs along different lines of sight. We have checked existing catalogues, and found that 25 QSOs are presently known with a redshift between $z=2-4$ and magnitude brighter than 16.5 . 


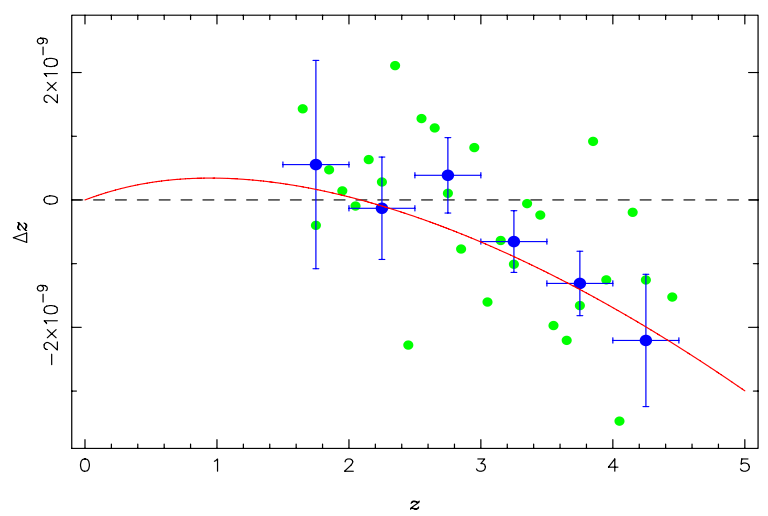

Figure 2. Monte Carlo simulation of a ź measurement using 30 pairs of Ly $\alpha$ forest spectra with $\mathrm{S} / \mathrm{N} 3000$ per pixel in the range $2<Z_{q s o}<5$. The solid line shows the expectation for the input cosmological model of $\mathrm{H}_{0}=70 \mathrm{~km} \mathrm{~s}^{-1} \mathrm{Mpc}^{-1}, \Omega_{m}=0.3$ and $\Omega_{d e}=0.7$

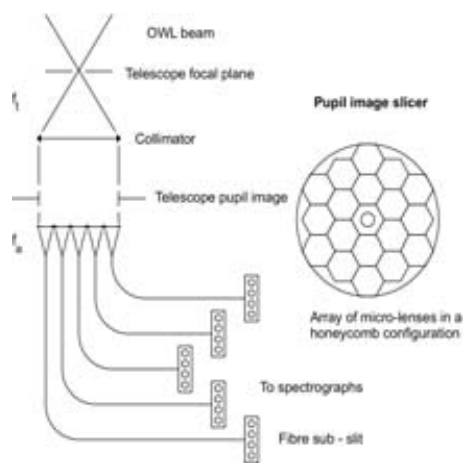

Figure 3. Slicing the pupil of the telescope. Each fibre bundle feeds one spectrograph.

\section{Instrument requirements and design}

Clearly we need a high resolution spectrograph working in the visible range with unprecedent capabilities of detecting tiny Doppler shifts. The wavelength range (400-700 $\mathrm{nm})$ precludes an effective use of $\mathrm{AO}$, so we studied a seeing limited instrument. A resolving power of 50000 would suffice to resolve the Ly $\alpha$ forest lines, but neither the associated metal lines would be resolved, nor an accurate wavelength calibration could be obtained. A compromise between resolving power, photon detection and feasibility has been found at $\mathrm{R}=150000$.

In order to obtain the required accuracy we opted for a super stable design, trying to improve the positive experience with HARPS (Mayor et al. (2003)). The concept is based on a number (5) of spectrographs nested in thermally and humidity controlled environments. To ensure proper light scrambling, a fibre system is required. We propose to slice the telescope pupil with a system of hexagonal minilenses, whose number will vary depending on the telescope diameter and aperture on the sky.

Figure 3 shows the slicing concept; the same slicer would work for an OWL telescope of $100 \mathrm{~m}$ diameter (with a 0.65 arcsecond aperture) or for a $60 \mathrm{~m}$ (with 1 arcsecond aperture). For the combination of the four VLT telescopes (1.4 arcsecond on the sky) only one spectrograph is required.

For an ELT the fibre length would exceed 200 meters, if the spectrograph was fed through the main focus. With such a length the absorption losses within the fibres become 
unacceptably high. CODEX requires an efficient way of feeding the spectrograph, while the fibres will be used only to scramble and slice the light.

\section{Other CODEX applications}

The characteristics and design of CODEX which are driven by the requirements for the main science case, will also be extremely beneficial for tackling a range of other important astrophysical questions. In the CODEX feasibility study we decided to concentrate on three highlight science cases for which CODEX offers the opportunity for a break through and which are well developed in the present literature. In addition to these selected applications, many other science cases emerged in the last years. Stellar oscillations, the study of the most metal poor stars in our Galaxy and in its local group companions, the use of cosmochronometers to determine the age of the Universe, the history of the metal enrichment of the Universe, the evolution of the CMB temperature with redshift. All these applications (and surely many more) will receive from CODEX@OWL an impressive boost, far beyond what can be now conceived.

\subsection{Variation of the fine structure constant $\alpha$}

The fundamental constants in physics albeit not deducable from first principles in the current established theoretical framework have long been believed by many to be universal and invariable quantities. This view is, however, increasingly challenged by recent theories of the Early Universe. Measuring time or spatial variations of one or more of the fundamental constants would have far reaching consequences for the unified theories of fundamental interactions, for the existence of extra dimensions of space and/or time and for the existence of scalar fields acting in the late universe. Astronomical observations offer a unique opportunity to probe the values of fundamental constants in the past, and in remote regions of space. With a spectrograph with $\mathrm{R} \sim 150000$ at OWL a gain in precision of two to three orders of magnitude with respect to present measurements is foreseen (cf. Molaro et al., these proceedings).

\subsection{Search for other earths}

Exo-planets and terrestrial planets in habitable zones will be one of the hottest topics of the next decades, and one of the main OWL science drivers. CODEX@OWL will lead the discoveries in at least three main cases in exo-planetary science, providingh unique capabilities and observations: i) discovery and confirmation of rocky planets, ii) search for long-period planets, iii) Jupiter mass planets around faint stars.

The need for a ground based follow-up facility capable of high radial velocity accuracy has been stressed in the recent ESO-ESA working group report on solar planets, which states as a high precision radial velocity instrumentation for the follow-up of astrometric and transit detections, is required, to ensure the detection of a planet by a second independent method, and to determine its true mass. For Earth-mass candidates, special purpose instrumentation (like HARPS) on a large telescope would be required.

As far as Hot Jupiters are concerned, CODEX will allow to search for these planets around solar mass stars in different environments and star forming histories, such as globular clusters and the nearby companions to the Galaxy.

\subsection{Primordial nucleosynthesis}

Standard Big Bang nucleosynthesis presents a pressing cosmological conundrum. There is some evidence suggesting a cosmological origin for ${ }^{6} \mathrm{Li}$ and the stellar value for primordial ${ }^{7} \mathrm{Li}$ does not agree with primordial D from QSOs and with WMAP. Although 
Li observations in low metallicity Galactic halo stars are plagued by possible systematic uncertainties due to modeling of stellar atmospheres and the treatment of convection, it is appealing that both the discrepancies can be reconciled with physics beyond the standard model during the Quark- Hadron phase. CODEX will allow first observations of ${ }^{7} \mathrm{Li}$ and ${ }^{6} \mathrm{Li}$ in dwarf stars in galaxies of the Local Group and it will make possible to measure for the first time the interstellar ${ }^{7} \mathrm{Li} /{ }^{6} \mathrm{Li}$ ratio in unprocessed material of High Velocity Clouds.

\section{Telescope requirements}

As explained above, the CODEX design is modular and can be adapted to a variety of telescope design and diameters.

(a) The strongest requirement is that CODEX needs to be located in an extremely stable (thermally and mechanically) environment.

(b) Since high efficiency is very important, a coudé train with CODEX-optimized coating should be made available.

(c) Without any scrambling, a guiding error of a few $\times 10^{-6}$ arcseconds would already lead to a radial velocity error of $1 \mathrm{~cm} \mathrm{~s}^{-1}$. While requirements on guiding can be relaxed by scrambling, it is still clear that the telescope must have excellent and reproducible centering and guiding capabilities.

(d) CODEX is designed as a seeing-limited instrument. AO would, however, help in concentrating the flux; fewer spectrographs would have to be built, or, alternatively, a higher fraction of the flux received. AO would likely help in improving the centering and guiding capabilities (cf. previous point).

\section{References}

Mayor, M. et al. 2003, The Messenger 114, 20

Pasquini, L. et al. 2005, The Messenger 122, 10

Perlmutter, S. et al. 1999, ApJ 517, 565

Riess, A.G. et al. 1998, AJ 116, 1009

Sandage, A. 1962, ApJ 136, 319

Spergel, D.S. et al. 2001, ApJS 148, 175 